\title{
FRECUENCIA DE LARVAS DIPHYLLOBOTHRIIDAE Y LARVAS ANISAKIDAE EN PECES MARINOS COMERCIALES DEL TERMINAL PESQUERO DE VENTANILLA-CALLAO
}

\author{
Cielo Llerena Z. ${ }^{1}$, Amanda Chávez V. ${ }^{2}$ y Eva Casas A. ${ }^{2}$
}

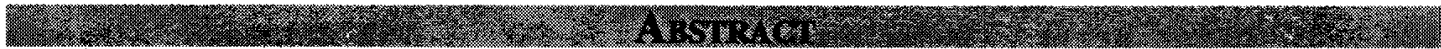

Frequency of Diphyllobothriidae and Anisakidae larva in commercial marine fish sold in the port of Callao.

The presence of the fish parasites Diphyllobothriidae and Anisakidae, as well as other helminthes capable of infecting marine mammals and human beings was evaluated in four commonly consumed species from the Peruvian coast. Muscle and abdominal cavity samples from 100 specimens each of caballa (Scomber japonicus), cachema (Cynoscion analis), coco (Paralonchurus peruanus) and lorna (Sciaena deliciosa), collected at the port of Callao from July to September 1999, were studied. Diphyllobothriidae plerocercoides larvae were found in $1 \%$ of the lorna, and Anisakidae larvae in $23 \%$ of the caballa, including Anisakis physeteris and A. simplex. Other larval forms found were Corynosoma obtuscens in 55\% of the lorna, $3 \%$ of the caballa, $16 \%$ of the coco and $2 \%$ of the cachema; Tentacularia coryphaenae in $4 \%$ of the caballa; and Nybelinia sp. in $13 \%$ of the lorna and $1 \%$ of the cachema. The helminthes were only found in the abdominal cavity. The heaviest parasite load was found in caballa, followed by lorna, and larval stages of Corynosoma obtuscens were found in all four salt water fish species.

Key words: Diphyllobothriidae, Anisakidae; Anisakis simplex; Anisakis physeteris; fish parasitism.

Los peces pueden presentar larvas plerocercoides de Diphyllobothriidae y larvas infectivas de Anisakidae capaces de ocasionar infecciones a mamíferos marinos y al hombre, asimismo pueden albergar otras formas larvarias que parasitan mamíferos marinos y algunos mamíferos terrestres. El objetivo de este trabajo fue determinar la frecuencia de larvas plerocercoides Diphyllobothriidae y larvas infectivas de la familia Anisakidae, así como la identificación y frecuencia de otros géneros de helmintos que pudieran hallarse en los peces muestreados, a nivel muscular y/o cavidad abdominal. Durante los meses de julio a setiembre de 1999, fueron muestreados 100 especímenes de cada una de las cuatro especies de peces marinos comerciales: caballa (Scomber japonicus); cachema (Cynoscion analis); coco (Paralonchurus peruanus) y lorna (Sciaena deliciosa) respectivamente, procedentes del terminal pesquero de VentanillaCallao, encontrándose larvas plerocercoides Diphyllobothriidae con frecuencia de $1 \%$ en lorna, larvas Anisakidae con $23 \%$ en caballa, de los cuales $20 \%$ correspondieron a Anisakis physeteris, y $4 \%$ a Anisakis simplex. Otras formas larvarias encontradas fueron Corynosoma obtuscens con $55 \%$ en lorna, $3 \%$ en caballa, $16 \%$ en coco y $2 \%$ en cachema; Tentacularia coryphaenae con 4\% en caballa; y Nybelinia sp. con $13 \%$ en lorna y $1 \%$ en cachema. Todos los helmintos fueron hallados sólo a nivel de cavidad

\footnotetext{
${ }^{1}$ Práctica privada

${ }^{2}$ Laboratorio de Parasitología IVITA-FMV-UNMSM. E-mail: d170010@unmsm.edu.pe
} 
abdominal. La caballa fue la especie con más carga parasitaria seguida de la lorna; mientras que la forma larvaria de Corynosoma obtuscens fue encontrada en las cuatro especies de peces marinos muestreados.

Palabras clave: Diphyllobothriidae, Anisakidae; Anisakis simplex; Anisakis physeteris; parasitismo en peces.

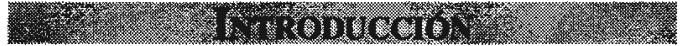

Se ha reportado que ciertos peces de consumo humano son portadores paraténicos o de transporte de ciertas formas larvarias parasitarias que tienen su desarrollo en el medio marino, pero que han sido descritas como patógenos potenciales para el humano, toda vez que se consuman pescados crudos (platos como "cebiche", "sushi", "sashimi"), ahumados o sometidos a cocción insuficiente y que alberguen la larva infectante (Atías, 1991). Tal es el caso de las enfermedades conocidas como difilobotriasis y anisakiasis, cuya forma larvaria denominada plerocercoide desarrolla a la forma adulta en el primer caso, y que para el segundo caso ocasiona parasitismo sin desarrollo de la forma adulta (Acha y Szyfres, 1989).

El presente estudio se realizó durante el periodo de julio a setiembre de 1999 con el objetivo de determinar la frecuencia de larvas de las familias Diphyllobothriidae y Anisakidae en los peces de la costa peruana.

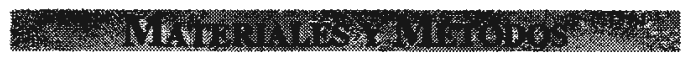

El estudio se realizó en 400 peces marinos de consumo humano, evaluándose 100 especímenes por cada especie: Scomber japonicus (caballa), Cynoscion analis (cachema), Paralonchurus peruanus (coco) y Scianea deliciosa (lorna) procedentes del terminal pesquero de Ventanilla-Callao. El reconocimiento de las características de los peces se basó en las descritas por Chirichigno (1998).

Las vísceras de los peces fueron examinadas in situ mediante un corte en la cavidad abdominal. Posteriormente se evaluaron los órganos por separado, y se observó el peritoneo parietal que recubre la cavidad celómica y la vejiga natatoria, realizándose cortes paralelos y delgados en los músculos hipoaxiales en búsqueda de quistes o formas larvarias de parásitos.

Las larvas halladas fueron lavadas en suero fisiológico y fijadas en alcohol al 70\%, para después ser medidas con regla milimétrica y/o ocular micrométrico. La identificación se hizo en base a características morfológicas descritas por Tantaleán (1972).

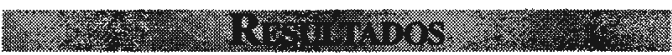

El Cuadro 1 muestra la frecuencia porcentual de larvas de las familias Diphyllobothriidae, larvas Anisakidae y otras familias de parásitos halladas en las cuatro especies de peces muestreados.

La única larva plerocercoide Diphyllobothriidae fue hallada en la especie lorna. Larvas infectivas de la familia Anisakidae solo fueron halladas en caballa. La larva plerocercoide Diphyllobothriidae fue hallada en un quiste de color blanco de $1.1 \mathrm{~mm} \times 1.2$ mm en la superficie de los intestinos. Las medidas de la larva fueron $120 \mu \mathrm{m}$ de longitud $\mathrm{x}$ $52.5 \mu \mathrm{m}$ de ancho.

Las larvas Anisakidae fueron halladas en la superficie de los intestinos además de la zona cercana al ano, en su mayoría en quistes de $2.0 \mathrm{~mm} \times 3.1 \mathrm{~mm}$ y en algunos casos se encontraron libres en la superficie de las vísceras. Las medidas de las larvas variaron entre 14.1 a $38.0 \mathrm{~mm}$ de longitud $\mathrm{x}$ 46.5 a $52.5 \mathrm{~mm}$ de ancho. 
Cuadro 1. Frecuencia de larvas de parásitos encontradas en 400 peces de 4 especies procedentes del Terminal Pesquero de Ventanilla-Callao.

\begin{tabular}{lcccccccc}
\hline Larvas Parásitas & \multicolumn{2}{c}{ Loma } & \multicolumn{2}{c}{ Caballa } & \multicolumn{2}{c}{ Coco } & \multicolumn{2}{c}{ Cachema } \\
\cline { 2 - 9 } & $\mathrm{N}^{\circ}$ & $\%$ & $\mathrm{~N}^{\circ}$ & $\%$ & $\mathrm{~N}^{\circ}$ & $\%$ & $\mathrm{~N}^{\circ}$ & $\%$ \\
\hline Fam Diphyllobothriidae & 1 & 1.0 & & & & & & \\
Fam Anisakidae & & & 23 & 23.0 & & & & \\
$\quad$ Anisakis simplex & & & 4 & 4.0 & & & & \\
$\quad \begin{array}{l}\text { Anisakis physeteris } \\
\text { Otras formas larvarias }\end{array}$ & & & 20 & 20.0 & & & & \\
$\quad \begin{array}{l}\text { Corynosoma obtuscens } \\
\text { Tentacularia coryphaenae }\end{array}$ & 55 & 55.0 & 3 & 3.0 & 16 & 16.0 & 2 & 2.0 \\
$\quad$ Nybelinia sp. & 13 & 13.0 & & & & & 1 & 1.0 \\
\hline
\end{tabular}

Formas larvarias del acantocéfalo Corynosoma obtuscens, fueron halladas en las cuatro especies muestreadas. Otro céstode como Tentacularia coryphaenae, fue hallado en caballa, y Nybelinia sp se pudo encontrar en lorna y cachema. Estas formas larvarias halladas fueron encontradas en la superficie del estómago y de los intestinos.

La presencia de formas larvarias de parásitos se ve influenciada por diversos factores que pueden ser cambios en la ecología marina como temperatura (muy ligada al crecimiento y desarrollo), humedad, luz, abundancia o disminución de hospederos intermediarios, hábitos alimenticios e inclusive tamaño del pez hospedero paraténico, el cual, a mayor tamaño, alberga mayor cantidad de larvas en sus músculos y vísceras (Atías, 1991).

Se sabe que la alimentación de los peces es uno de los factores que influye en la presentación de larvas parasitarias en los peces hospederos paraténicos, así tenemos que la lorna suele vivir en los fondos arenosos en las cercanías de las playas, donde se alimenta de pequeños crustáceos y organismos relacionados con el fondo marino, pero también incluye en su dieta pequeños peces que pueden contener la forma larvaria plero- cercoide Diphyllobothriidae. La caballa, basa su alimentación principalmente en el consumo de anchoveta, y pequeños crustáceos, razón por la cual posiblemente sea la especie más infectada de larvas parásitas de las cuatros especies estudiadas en este trabajo. La cachema, consume una dieta mixta prefiriendo los organismos del fondo marino, y es pocas veces carnívoro, es decir, tiene menos posibilidad de consumir hospederos intermediarios de larvas parásitas. La especie coco consume organismos del fondo marino, con patrones de comportamiento aún desconocidos. Esta especie tiene una presentación predominante de ectoparásitos y la ausencia casi total de formas larvarias de helmintos transmisibles como Diphyllobothriidae, Anisakidae, acantocéfalos, que son parásitos comunes de los peces marinos (IMARPE, 1998; Luque y Oliva, 1993).

En el presente estudio se obtuvo una frecuencia del $1 \%$ para larvas Diphyllobothriidae de un total de 100 lornas, sin poder encontrar en las demás especies muestreadas. En estudios similares realizados en 1972 con peces provenientes del terminal del Callao y del área de Chimbote, se halló una frecuencia de $6.5 \%$ para lorna y $0.5 \%$ para coco (Tantaleán, 1975). Otro estudio realizado durante los meses de abril a junio de 1997 se obtuvo 4.29\% de larvas Diphyllobothriidae en jurel (Pérez, 1995). 
Asimismo se obtuvo una frecuencia del $23 \%$ para larvas anisakidae de un total de 100 caballas, no encontrándose dicha forma larvaria en el resto de las especies sometidas a muestreo. En trabajos similares, con peces procedentes del terminal pesquero del Callao, se obtuvo una frecuencia de $\mathbf{4 8 . 6 \%}$ en 22 jureles, $16 \%$ en 180 cocos, $1.5 \%$ en 381 lornas (Tantaleán, 1972), mientras que en 1997 se obtuvo una frecuencia de $8.5 \%$ de 70 jureles, $47.1 \%$ de 70 bonitos y $20 \%$ de 70 lisas (Pérez, 1998).

Las formas larvarias del acantocéfalo Corynosoma obtuscens halladas en nuestro estudio en el 55, 3, 16 y 2 de especímenes de lorna, caballa, coco y cachema, respectivamente, han sido descritas parasitando en su forma adulta a mamíferos marinos y a perros del departamento de Ica (Rojas et al., 1997), mientras que las larvas de céstodes como Tentacularia coryphaenae y Nybelinia $s p$. que fueron halladas en diversos porcentajes, han sido descritas parasitando tiburones y rayas (Tantaleán y Huiza, 1994). La presencia de estas formas larvarias se debe probablemente al hábito alimenticio de las especies de peces muestreados, como es el de consumir pequeños crustáceos.

De las cuatro especies estudiadas, la caballa constituye la especie más parasitada, seguida de la lorna, en la que se encontraron diferentes tipos de formas larvarias en diversos porcentajes.

La forma larvaria de Corynosoma obtuscens fue hallada en las cuatro especies de peces marinos muestreados, constituyendo un riesgo para mamíferos terrestres que consumen estos peces infectados.

- Realizar campañas de difusión a la población acerca de la enfermedad y el riesgo potencial que significa consumir pescado crudo o mal cocido infectado con estos tipos de larvas. Asimismo, recomendar el consumo de pescado fresco, rápidamente eviscerado.

- Revisar el pescado al realizar su compra, observando si existen residuos de vísceras que puedan presentar los quistes, que son fácilmente visibles.

1. Acha, N. P. y B. Szyfres. 1989. Zoonosis y enfermedades transmisibles comunes al hombre y a los animales. 2da. ed., Editorial OPS/OMS. E.U.A, p. 989.

2. Atías, A. 1991. Parasitología Clínica. En Carbajal J. Anisakiasis; Torres y Pérez C. Difilobotriasis 3ra. ed., p.190193, 203-212. Editorial Mediterráneo. Chile.

3. Chirichigno, F. N. 1998. Clave para identificar los peces marinos del Perú. Publicación especial. 2da. ed; 44:412435. IMARPE. Perú.

4. IMARPE. 1998. Áreas de pesca de la flota artesanal de la Caleta Santa Rosa, Chiclayo-Perú 1996-1998. Informe $\mathrm{N}^{\circ}$ 142. Dic:25-33.

5. Luque, L. J. y M. Oliva. 1993. Análisis cuantitativo y estructura de la comunidad parasitaria de Paralonchurus peruanus (Pisces:Scianidae) en la costa peruana. Parasitol al Día 17:107-111.

6. Pérez, I. 1998. Frecuencia de larvas plerocercoides de Diphyllobothriidae y larvas infectivas de Anisakidae en cinco especies de peces comerciales de Lima Metropolitana. Tesis Méd. Vet. Fac. Med. Vet. Univ. Nac. Mayor San Marcos, Lima. 107p.

7. Rojas, R., M. Dávalos y R. Cabrera. 1997. Prevalencia de Corynosoma obtuscens (Acanthocephala) en Canis familiaris de las provincias de Chincha 
y Pisco. En III Congreso Peruano de Parasitología, Arequipa- Perú. Set 1719: 82.

8. Tantaleán, M. 1972. La presencia de larvas Anisakis sp en algunos peces del mar peruano. Rev. Per. Méd. Tropical. UNMSM 11(1): 38- 43.
9. Tantaleán, M. 1975. Hallazgo de larvas plerocercoides de Diphyllobothriidae en peces del mar peruano. Bol. Chile. Parasit. 30:18-20.

10. Tantaleán, M. y A. Huiza. 1994. Sinopsis de los parásitos marinos de la costa peruana. Biotempo. UNMSM 1:53-101. 